\title{
Invasive Klebsiella pneumoniae Syndrome in Qatar: A Case Report
}

\author{
Muhammad B. Jamshaid ${ }^{1}$, Aamir Shahzad ${ }^{2}$, Abdulaziz Zafar ${ }^{3}$, Ijaz Kamal ${ }^{3}$ \\ 1. Internal Medicine, Hamad General Hospital, Doha, QAT 2. Medicine, Hamad General Hospital, Doha, QAT 3. Internal \\ Medicine, Hamad Medical Corporation, Doha, QAT
}

Corresponding author: Muhammad B. Jamshaid, drbilaljamshaid@gmail.com

\begin{abstract}
Klebsiella pneumoniae (K. pneumoniae) is a Gram-negative bacteria that can infect most of the body's organs, from the lungs to the central nervous system. It is notorious for causing pneumonia in alcoholic, diabetic, and hospitalized patients. It is now emerging as a cause of abscesses involving multiple organs. Invasive $K$. pneumoniae is most commonly observed in the Asian population but has been reported in other geographical areas as well. We present a case of invasive $K$. pneumoniae. The patient was initially admitted with diabetic ketoacidosis (DKA); further investigations showed multiple abscesses involving the liver, lungs, brain, and muscles. K. pneumoniae was identified in blood and liver abscess cultures. The patient was managed for DKA as per protocol, and was administered broad-spectrum antibiotics with percutaneous drainage of liver abscess for invasive $K$. pneumoniae syndrome. In this paper, we highlight the invasive nature of K. pneumoniae, which may aid clinicians in diagnosing and managing similar cases, thereby preventing the associated high morbidity and mortality.
\end{abstract}

Categories: Internal Medicine, Infectious Disease

Keywords: diabetic ketoacidosis (dka), klebsiella pneumonea, disseminated bacteremia, liver abscess aspiration, brain abscess

\section{Introduction}

Klebsiella pneumoniae (K. pneumoniae) is a Gram-negative encapsulated bacillus that is widely present in nature, including the human oral cavity and intestine [1]. K. pneumoniae is the most important organism in the Klebsiella genus; it is responsible for hospital-acquired infections in compromised hosts with impaired immune systems. Infections caused by K. pneumoniae can be acquired in long-term care facilities, such as nursing homes, and less often, in the community. It can cause various infections, including hospital- and community-acquired pneumonia, bloodstream infections, lung abscesses, empyema, bacteremia, catheterrelated infections, wound or surgical site infections, upper and lower urinary tract infections, liver abscesses, and meningitis [2].

Review began 04/30/2021 Review ended 05/07/2021 Published 05/13/2021

\section{(๑) Copyright 2021}

Jamshaid et al. This is an open access article distributed under the terms of the Creative Commons Attribution License CC-BY 4.0., which permits unrestricted use, distribution, and reproduction in any medium, provided the original author and source are credited.
K. pneumoniae infections occur particularly in patients with chronic alcoholism, diabetes, chronic kidney disease, and those on long-term steroids. Less common infections include endophthalmitis, psoas muscle abscesses, septic arthritis, pyomyositis, and purulent pericarditis [3]. Multiorgan infections occur from an embolic spread of invasive liver abscesses. Embolic Klebsiella infection appears to be a rare complication and accounts for only $2 \%$ of the total cases [4]. However, it may lead to high morbidity and mortality [5].

\section{Case Presentation}

A 40-year-old Nepalese male was brought to the emergency department (ED) by emergency medical services (EMS) due to an episode of acute confusion, headache, and dizziness. He had been recently diagnosed with poorly controlled type 2 diabetes mellitus (HbA1c: 13.6). On examination, the patient was vitally stable; he was aggressive, disoriented to time and place, and had a low level of consciousness, which led to the patient's intubation in the ED. The emergency head CT was unremarkable. On review of his blood investigations, he was found to have acidosis with a PH of 6.9 (normal range: 7.34-7.45), bicarbonate of 6 $\mathrm{mmol} / \mathrm{L}$ (normal range: $24-28 \mathrm{mmol} / \mathrm{L}$ ), and elevated serum beta-hydroxybutyrate level of 6.6 $\mathrm{mmol} / \mathrm{L}$ (normal value: $<0.4 \mathrm{mmol} / \mathrm{L}$ ). Based on these results, treatment was started for diabetic ketoacidosis (DKA) with IV fluids and IV insulin as per local protocol, and the patient was admitted to the medical intensive care unit (MICU) for further management.

Further investigations showed elevated inflammatory markers with leukocytosis. His WBC was $15 \times 10^{3} / \mathrm{uL}$ (normal range: $4-10 \times 10^{3}$ ) and C-reactive protein (CRP) was $320 \mathrm{mg} / \mathrm{L}$ (normal level: $<6 \mathrm{mg} / \mathrm{L}$ ). After taking samples of blood and urine for culture, the administration of empiric antibiotics piperacillin-tazobactam and clarithromycin were started. A chest X-ray (Figure 1) showed patchy opacities in both lung fields. Later, K. pneumoniae was identified in both the blood culture and the tracheal aspirate culture. Antibiogram showed good sensitivity to multiple antibiotics, and hence antibiotic treatment was de-escalated to amoxicillin-clavulanate, as per the local infectious disease protocol. 


\section{Cureus}

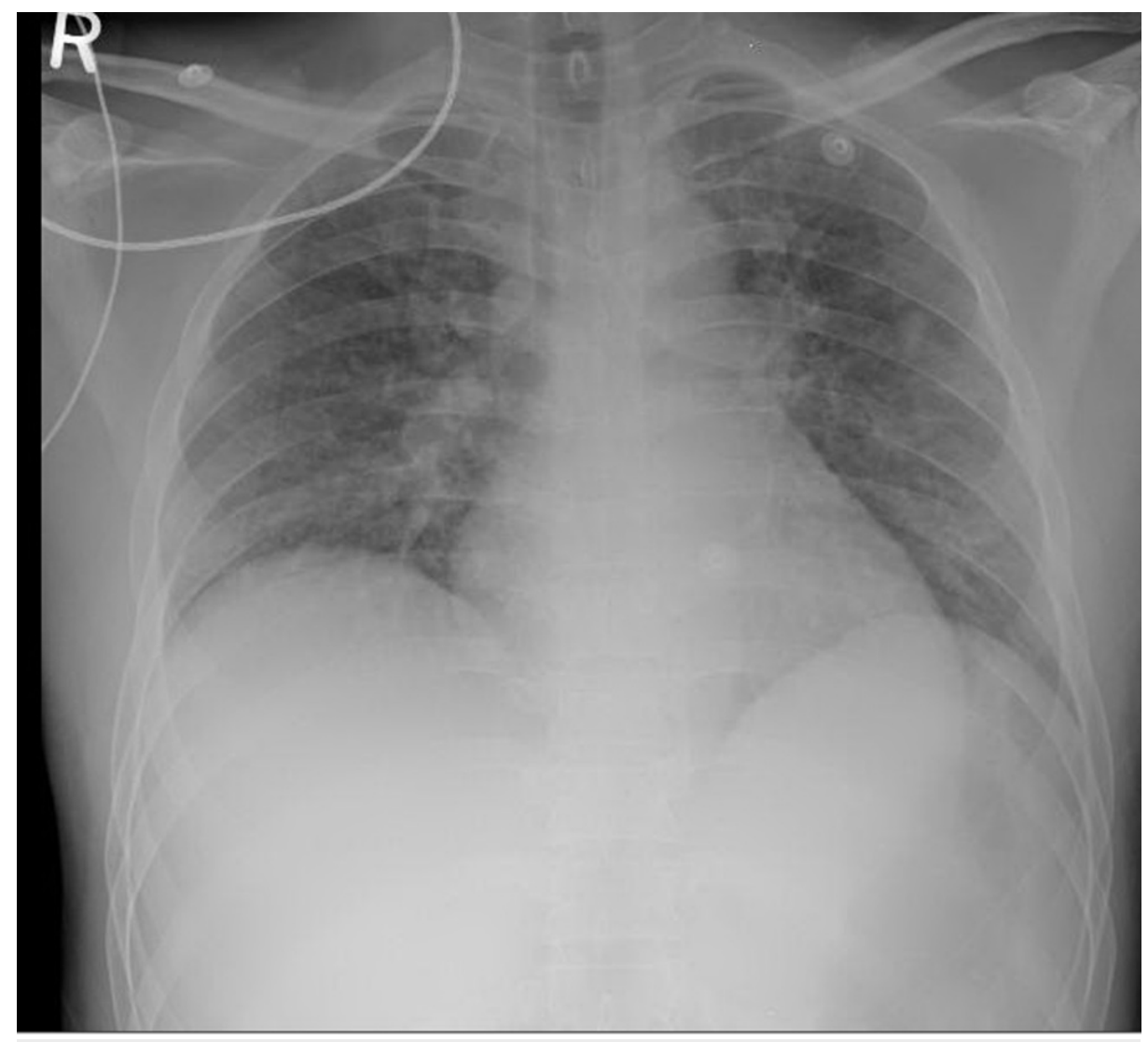

FIGURE 1: Chest X-ray showing bilateral fluffy infiltrates

On day three, the patient's condition deteriorated as he got hypotensive, requiring inotropic support. An abdominal ultrasound scan revealed an enlarged liver of $17.7 \mathrm{~cm}$ and an ill-defined complex lesion of $6.3 \times 6$ x $5.1 \mathrm{~cm}$, with radiological findings indicating hepatic abscess (Figure 2A).

An abdominal CT confirmed the presence of abscesses. It showed an abscess in the liver, multiple small abscesses in both kidneys, and numerous air pockets of abscesses in the right gluteus (Figure 3). During the same day, the patient developed anisocoria. The initial non-contrast head CT was unremarkable. A head MRI showed septic embolic meningoencephalitis with widespread microhemorrhages (Figure 4A). Ultrasoundguided drainage of the liver abscess was performed, and the drained fluid culture was also positive for $K$. pneumoniae. The antibiotics were escalated to meropenem, vancomycin, and metronidazole by the infectious disease team. Transthoracic echocardiogram and transesophageal echocardiogram ruled out any heart valve vegetations. All these tests confirmed that the patient had an embolic spread of Klebsiella infection.

On day 10 , the patient became hemodynamically stable, which led to the tapering of inotropes and sedation. Despite the administration of multiple antibiotics, the patient continued to have a high-grade fever. An abdominal ultrasound performed 15 days after the ultrasound-guided liver drainage showed interval regression of the liver abscess's size to $4.4 \times 4.8 \times 4 \mathrm{~cm}$ and an estimated abscess volume of $54 \mathrm{cc}$ (Figure 2B).

After receiving antibiotics for two weeks, the patient's culture was found to be negative for K. pneumoniae. The patient became afebrile and hemodynamically stable, and antibiotics were de-escalated to ceftriaxone and metronidazole. However, the patient's Glasgow Coma Scale score remained low, and the follow-up head MRI showed a worsening of microabscesses (Figure 3B). Therefore, a tracheostomy was performed, and the patient continued to receive tracheostomy ventilation.

Ceftriaxone and metronidazole were administered for a further six weeks. The patient remained afebrile during this period, and he was shifted to a long-term facility for continuity of care. 


\section{Cureus}

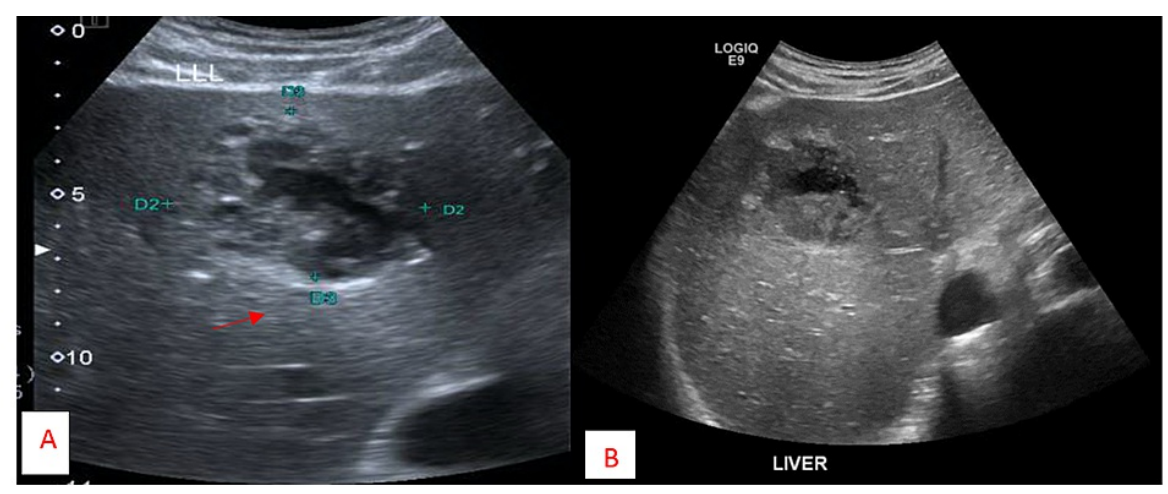

\section{FIGURE 2: Ultrasound of the abdomen}

A. Ultrasound of the abdomen showing abscess of dimensions $6.3 \times 6 \times 5.1 \mathrm{~cm}$ and estimated volume of 94 cc. B. Repeat ultrasound performed 15 days after ultrasound-guided abscess drain showing regression in the liver abscess of dimensions $4.4 \times 4.8 \times 4 \mathrm{~cm}$ and estimated volume of $54 \mathrm{cc}$
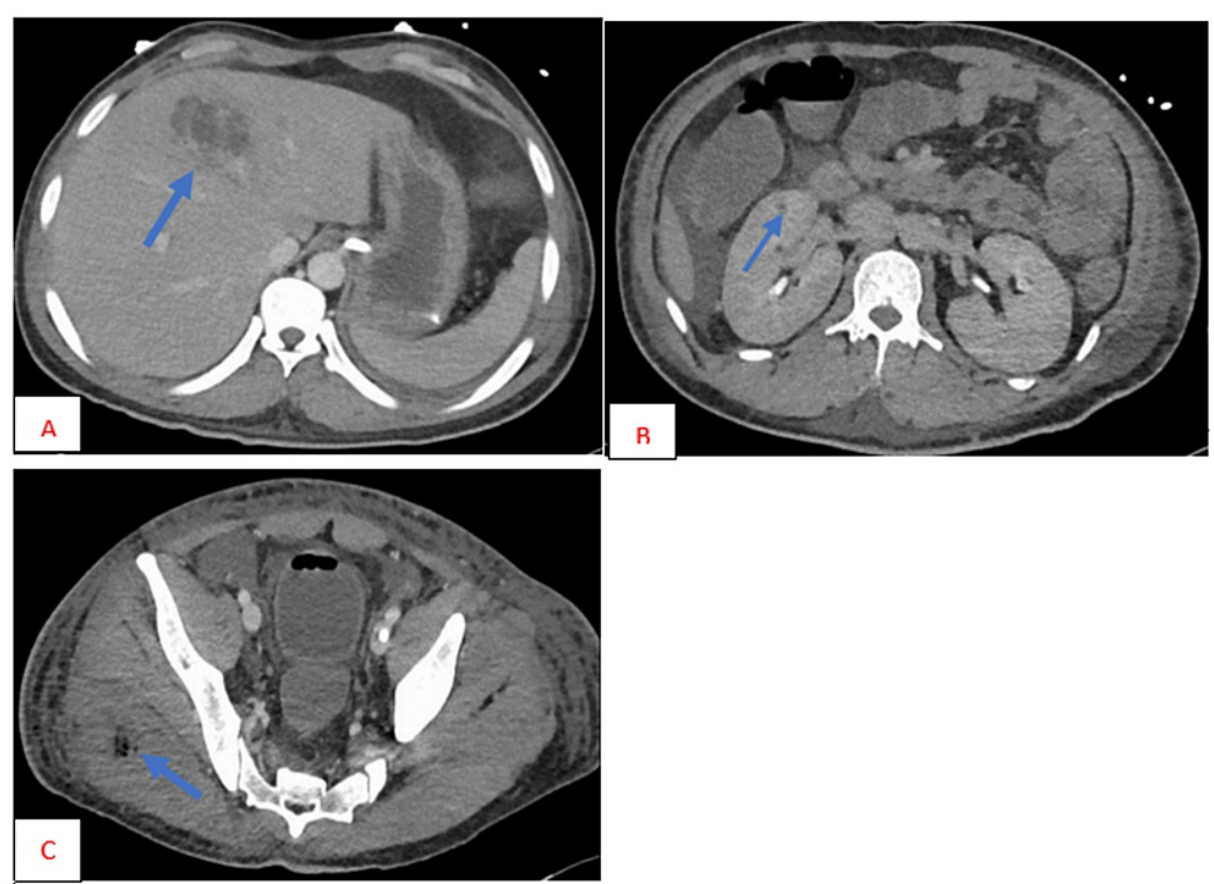

FIGURE 3: CT of the abdomen

The images showing (A) liver abscess, (B) kidney abscess, and (C) gluteal muscle abscess (blue arrows)

CT: computed tomography 


\section{Cureus}

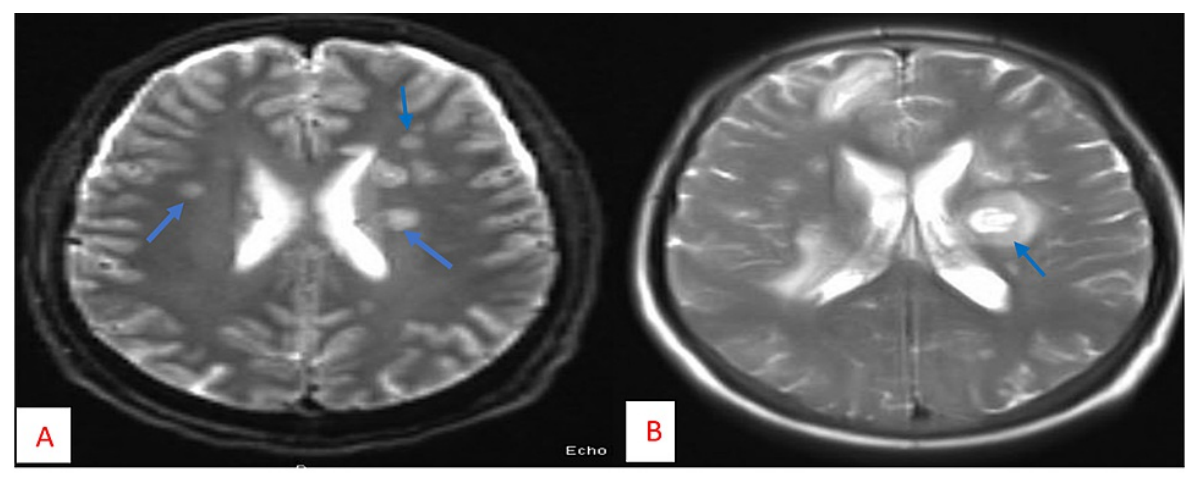

\section{FIGURE 4: MRI of the head}

A. First T1Wi MRI head showing bilateral cerebral abscess. B. Repeat T1Wi MRI head 20 days after the first MRI showing new lesions

MRI: magnetic resonance imaging

\section{Discussion}

Carl Friedlander first identified $K$. pneumoniae in a lung autopsy of a patient who had died from pneumonia in 1882. The bacterium was initially named Friedlander's bacillus. K. pneumoniae is considered to be the most common cause of hospital-acquired pneumonia in the United States, and it also accounts for 3-8\% of nosocomial infections [6]. The pathogenicity of $K$. pneumoniae depends not only on host factors such as diabetes or chronic alcoholism but also on bacterial virulence [7]. Several virulence factors contribute to the pathogenicity, including hypermucoviscous-specific capsular antigens (i.e., K1 and K2 serotypes) and virulence genes FimH (fimbrial adhesion), rmpA (regulator of mucoid phenotype), uge (uridine-diphosphate galacturonate 4-epimerase), kfu (an iron uptake system), and alls (allantoin metabolism). The K1 and K2 hypermucoviscous-specific capsular serotypes are associated with invasive infections and usually have poor prognoses [7-10].

In our case of embolic K. pneumoniae, the risk factor was uncontrolled diabetes mellitus, which is well described in the literature and is very common in the Middle East. Therefore, the Middle East is a particularly high-risk area for $K$. pneumoniae infection, and this issue needs urgent attention. Clinicians must be vigilant to diagnose and manage it effectively and efficiently.

The prognosis for K. pneumoniae liver abscess (KLA) is generally good, but embolic complications can cause significant morbidity [7], as in our case, where severe brain damage occurred. KLA with the embolic disease has been predominantly observed in Asia, particularly in Taiwan, but there are case reports from other geographical regions as well [11].

Hypervirulent strains of K. pneumoniae can cause a destructive tissue abscess syndrome (e.g., primary liver abscess) with possible metastatic infection. These strains are generally susceptible to cephalosporins, although resistant strains, including those that produce an extended-spectrum $\beta$-lactamase (ESBL) or a carbapenem, have been reported before. Appropriate antimicrobial treatment combined with percutaneous drainage of liver abscesses increases the chances of survival for such patients. However, the availability of a facility for early detection of the virulent strain will help in early diagnosis and treatment, which will reduce morbidity and improve outcomes [5].

\section{Conclusions}

We discussed the case of a patient with invasive $K$. pneumoniae. The presentation of $K$. pneumoniae can range from pneumonia to disseminated infection. It should be considered in the differential diagnosis of patients presenting with multiorgan abscesses.

\section{Additional Information}

\section{Disclosures}

Human subjects: Consent was obtained or waived by all participants in this study. Medical Research Center at Hamad Medical Corporation issued approval 04-20-911. Medical Research Center at Hamad Medical Corporation has granted permission for this case report to be published on the condition that no patientidentifiable data (including patient name and photograph) are included. Conflicts of interest: In compliance with the ICMJE uniform disclosure form, all authors declare the following: Payment/services info: All authors have declared that no financial support was received from any organization for the 
submitted work. Financial relationships: All authors have declared that they have no financial relationships at present or within the previous three years with any organizations that might have an interest in the submitted work. Other relationships: All authors have declared that there are no other relationships or activities that could appear to have influenced the submitted work.

\section{References}

1. CDC: Healthcare-associated Infections (HAI): Klebsiella pneumoniae in healthcare settings . (2010). Accessed: August 30, 2020: https://www.cdc.gov/hai/organisms/klebsiella/klebsiella.html.

2. Klebsiella pneumoniae. (2020). Accessed: August 30, 2020: https://www.sciencedirect.com/topics/medicineand-dentistry/klebsiella-pneumoniae.

3. Shi YF, Wang YK, Wang YH, Liu H, Shi XH, Li XJ, Wu BQ: Metastatic infection caused by hypervirulent Klebsiella pneumonia and co-infection with Cryptococcus meningitis: a case report. World J Clin Cases. 2019, 7:3812-20. 10.12998/wjcc.v7.i22.3812

4. Fang CT, Lai SY, Yi WC, Hsueh PR, Liu KL, Chang SC: Klebsiella pneumoniae genotype K1: an emerging pathogen that causes septic ocular or central nervous system complications from pyogenic liver abscess. Clin Infect Dis. 2007, 45:284-93. 10.1086/519262

5. Siu LK, Yeh KM, Lin JC, Fung CP, Chang FY: Klebsiella pneumoniae liver abscess: a new invasive syndrome . Lancet Infect Dis. 2012, 12:881-7. 10.1016/S1473-3099(12)70205-0

6. Fang CT, Chuang YP, Shun CT, Chang SC, Wang JT: A novel virulence gene in Klebsiella pneumoniae strains causing primary liver abscess and septic metastatic complications. J Exp Med. 2004, 199:697-705. 10.1084/jem.20030857

7. Cheng DL, Liu YC, Yen MY, Liu CY, Wang RS: Septic metastatic lesions of pyogenic liver abscess. Their association with Klebsiella pneumoniae bacteremia in diabetic patients. Arch Intern Med. 1991, 151:1557-9.

8. Evangelista V, Gonçalves CV, Almeida R, Henriques C, Baptista AM, da Graça JP, Araújo JL: Klebsiella pneumoniae invasive syndrome. Eur J Case Rep Intern Med. 2018, 5:000800. 10.12890/2018_000800

9. Xu M, Li A, Kong H, Zhang W, Chen H, Fu Y, Fu Y: Endogenous endophthalmitis caused by a multidrugresistant hypervirulent Klebsiella pneumoniae strain belonging to a novel single locus variant of ST23: first case report in China. BMC Infect Dis. 2018, 18:669. 10.1186/s12879-018-3543-5

10. Chiu HHC, Francisco CN, Bruno R, Jorge Ii M, Salvaña EM: Hypermucoviscous capsular 1 (K1) serotype Klebsiella pneumoniae necrotising fasciitis and metastatic endophthalmitis. BMJ Case Rep. 2018, 11:e226096. 10.1136/bcr-2018-226096

11. Sobirk SK, Struve C, Jacobsson SG: Primary Klebsiella pneumoniae liver abscess with metastatic spread to lung and eye, a north-European case report of an emerging syndrome. Open Microbiol J. 2010, 4:5-7. $10.2174 / 1874285801004010005$ 